\section{NTA NEWS}

\section{Ankündigung}

ITA-Jahreskonferenz „TA'16 - Smart New World“"

\section{Smart New World - Was ist smart an „smarten“ Technologien?}

\section{Mai 2016}

Österreichische Akademie der Wissenschaften, Dr. Ignaz Seipel-Platz 2, 1010 Wien, Österreich; http://www.oeaw.ac.at/ita/ta16

Smart Cities sollen durch neue Technologien effizient und umweltfreundlich werden. Smart Homes verheißen durch intelligente, also Daten sammelnde und verarbeitende Geräte und Steuerungssysteme bessere Anpassung an unsere Bedürfnisse. Smart Grids sollen die Flexibilität der Energieversorgung erhöhen, Smart Cars uns das Steuern von Fahrzeugen abnehmen, und Smart Production soll als Industrie 4.0 eine neue Epoche industriellen Produzierens einleiten. Die Digitalisierung aller Lebensbereiche bringt weitgehende Veränderungen. Eines haben sie alle scheinbar gemeinsam: All diese technischen Visionen versprechen unser Leben besser - bequemer, effizienter, sicherer, bedürfnisgerechter, ressourcenschonender oder gesünder - zu machen.

Ermöglicht uns das Label „smart“ ein Mehr an Autonomie, oder laufen wir dabei Gefahr, durch Algorithmen fremdbestimmt zu werden? Führt uns die steigende Tendenz zur Selbstoptimierung in die Isolation des Einzelnen? Die vom Institut für Technikfolgen-Abschätzung (ITA) der Österreichischen Akademie der Wissenschaften veranstaltete Konferenz widmet sich einer kritischen Auseinandersetzung mit smarten Technologien und deren scheinbar universellen Anwendungsbereichen. Smarte Technologien“ verändern nicht nur die Beziehung zwischen Mensch und Technik, sie stellen auch grundlegende gesellschaftliche Werte wie Privatsphäre oder Autonomie zur Debatte. Die Keynote-Vorträge kommen von Dirk Helbing, Professor für Soziologie an der ETH Zürich, und Sarah Spiekermann, Leiterin des „Institute for Management Information Systems" an der Wirtschaftsuniversität Wien.

Kontakt: tamail@oeaw.ac.at

\section{Erinnerung}

\section{NTA-Konferenz in Bonn}

Grand Challenges meistern - der

Beitrag der Technikfolgenabschätzung

16.-18. November 2016

\section{Universitätsclub Bonn}

Call for Papers: http://www.ea-aw.de/nta7-2016

Grand Challenges spielen derzeit eine rahmende Rolle für die Förderung und Koordinierung gesellschaftlich relevanter Forschung und Innovation in Europa. Konkret werden damit neben dem demografischen Wandel spezifische Herausforderungen durch Klimawandel, Gesundheitsfragen, moderne Mobilitäts- und Kommunikationskonzepte sowie für die zivile Sicherheit erkannt. Dabei gilt es, die Entwicklung geeigneter Schlüsseltechnologien oder Verfahrensansätze im gesellschaftlichen Dialog zu diskutieren, zu entwickeln und zu fördern. Grand Challenges sind somit auch Gegenstände von Technikreflexion und -abschätzung. Hierzu zählen die Beurteilung technisch induzierter Risiken, die Reflexion von nachgelagerten Nachhaltigkeits- und Akzeptanzfragen und entsprechende Lösungsvorschläge aus technikethischer sowie aus innovationsgetriebener Perspektive.

Auf der NTA-Konferenz werden Vortragende und Plenum aus Sicht der TA Optionen, Potenziale und Barrieren sowie mögliche Nachteile einer an Grand Challenges orientierten Forschungs- und Innovationspolitik erörtern. Die Konferenz wird mit einer Podiumsdiskussion zur Bedeutung von Grand Challenges für die Politikberatung eingeleitet. Spezifische Fragen zum Thema sollen dann im Rahmen vertiefender Fachsitzungen erörtert werden. Vorschläge für entsprechende Präsentationen oder Poster können bis zum 30. Mai 2016 eingereicht werden.

Die Organisation der Konferenz liegt bei der EA European Academy of Technology and Innovation Assessment, die hiermit auch ihr 20-jähriges Bestehen feiert. Die Tagung wird vom DLR, Fraunhofer ISI, ITA, ITAS, der TA SWISS und der Universität Bielefeld unterstützt.

Kontakt: Dr. Stephan Lingner; stephan.lingner@ea-aw.de 